\title{
Risk of infection in patients with lymphoma receiving rituximab: systematic review and meta-analysis
}

\author{
Simone Lanini* ${ }^{*}$, Aoife C Molloy ${ }^{2}$, Paul E Fine ${ }^{3}$, Archibald G Prentice ${ }^{4}$, Giuseppe Ippolito ${ }^{1}$ and \\ Christopher $C$ Kibbler $^{2}$
}

\begin{abstract}
Background: The addition of Rituximab (R) to standard chemotherapy (C) has been reported to improve the end of treatment outcome in patients affected by CD-20 positive malignant lymphomas (CD20+ ML). Nevertheless, given the profound and prolonged immunosuppression produced by $\mathrm{R}$ there are concerns that severe infections may arise. A systematic review and meta-analysis were performed to determine whether or not the addition of $R$ to $C$ may increase the risk of severe infections in adults undergoing induction therapy for CD20+ ML.

Methods: Only randomised controlled trials comparing R-C to $C$ standard alone in adult patients with CD20+ ML were included. Meta-analysis was performed on overall incidence of severe infection, risk of dying as the consequence of infection, risk of febrile neutropenia, risk of severe leucopenia, risk of severe granulocytopenia and overall response assuming a fixed effect model. Heterogeneity was investigated, if present and $\mathrm{I}^{2}>20 \%$, according to several predefined baseline characteristics of the study populations.
\end{abstract}

Results: Several relevant results have emerged. First, the addition of $\mathrm{R}$ to standard $\mathrm{C}$ does not increase the overall risk of severe infections ( $R R=1.00 ; 95 \% \mathrm{Cl} 0.87$ to 1.14) nor does it increase the risk of dying as a consequence of infection ( $R R=1.60 ; 95 \% \mathrm{Cl} 0.68$ to 3.75). Second, we confirmed that the addition of $\mathrm{R}$ to standard $\mathrm{C}$ increases the proportion of overall response ( $R R=1.12 ; 95 \% \mathrm{Cl} 1.09$ to 1.15 ), but it also increases the risk of severe leucopenia $(\mathrm{RR}=1.24 ; 95 \% \mathrm{Cl} 1.12$ to 1.37$)$ and granulocytopenia ( $\mathrm{RR}=1.07 ; 95 \% \mathrm{Cl} 1.02$ to 1.12$)$.

Conclusions: $\mathrm{R}-\mathrm{C}$ is superior to standard $\mathrm{C}$ in terms of overall response and it does not increase the overall incidence of severe infection. However, data on special groups of patients (for example, HIV positive subjects and HBV carriers) are lacking. In our opinion more studies are needed to explore the potential effect of $\mathrm{R}$ on silent and chronic viral infections.

\section{Background}

CD20 positive $(\mathrm{CD} 20+)$ malignant lymphomas $(\mathrm{ML})$ are a group of potentially lethal neoplasms with an incidence rate of approximately 19 cases per 100,000 person-years in Europe and represent one of the leading causes of cancer in adults [1]. The last revision of the World Health Organization (WHO) "Classification of Tumours of Haematopoietic and Lymphoid Tissues" identified 40 CD-20+ ML subtypes [2]. From a practical

\footnotetext{
* Correspondence: simone.lanini@inmi.it

'National Institute for Infectious Diseases, INMI-Lazzaro Spallanzani Via Portuense, 29200149 Rome, Italy

Full list of author information is available at the end of the article
}

point of view the different histological subtypes can be grouped according to their clinical features, into aggressive, potentially curable, and indolent, as yet incurable. The majority of CD20+ ML in adults are indolent lymphomas and include different histological subtypes such as chronic lymphocytic leukemia/small lymphocytic lymphoma (CLL), follicular lymphoma (FL), marginal zone lymphoma (MZL) and lymphoplasmacytic lymphoma (LPL). Aggressive lymphomas are less common in adults and include diffuse large B-cell lymphomas (DLBCL) and all HIV-associated lymphomas.

Effective multi-drug chemotherapy (C) protocols for CD20+ ML have been available for the last 30 years

\section{() Biomed Central}


with variable results in indolent and aggressive ML but protocols have been changing recently with the introduction of rituximab ( $R$ ) [3]. $R$ is a chimerical anti$\mathrm{CD} 20$ monoclonal antibody (MoAb) with activity against normal and malignant B-cells expressing the cell-surface molecule CD20. Recent systematic reviews provide evidence that, in comparison to $\mathrm{C}$ alone, the combination of $\mathrm{R}$ and $\mathrm{C}(\mathrm{R}-\mathrm{C})$ may increase the remission both in indolent [4] and aggressive CD20+ ML [5,6]. However, given the profound and prolonged immunosuppression produced by $\mathrm{R}$, there are concerns that infections may arise [7].

In studies of HIV positive (HIV+) patients with ML there is evidence of an increased risk of infections when $\mathrm{R}$ is added to $\mathrm{C}$, in particular for patients with CD4 counts less than 50 cells $/ \mathrm{ml}$ [8]. In pooled results from three phase II trials of patients with ML receiving $\mathrm{R}-\mathrm{C}$, $31 \%$ of patients developed severe infections, compared with $20 \%$ incidence reported in prior studies with similar $\mathrm{C}$ protocols [9]. In the only phase III trial published so far, comparing R-C to $\mathrm{C}$ in HIV-associated ML, the mortality due to infection was significantly higher in R$C$ than in standard C: $14 \%$ and $2 \%$ respectively $(P=$ 0.035) [8].

A meta-analysis comparing $\mathrm{R}$ maintenance therapy with observation in HIV negative subjects indicated that the risk of infections in the intervention was double that in the control arm [10].

Case reports and case series also suggest that $R$ increases the risk of viral infections [11]. Potentially lethal reactivations of hepatitis B virus (HBV) [12] may occur after $\mathrm{R}$ therapy both in patients with resolved $\mathrm{HBV}$ (anti-HBsAg+) and in those with isolated HBV core antibody positivity (anti-HBc+) $[13,14]$. These observations are strengthened by other reports which show that protective antibody against HBV (antiHBsAg) may diminish, or even vanish, soon after $R$ administration in some patients $[15,16]$ and that patients receiving anti-HBV prophylaxis during $\mathrm{R}-\mathrm{C}$ regimens may experience no reactivation of their latent HBV infection [14-17].

Problems with other viruses have also been reported in association with R-containing regimens. Severe herpes virus reactivation (for example, cytomegalovirus [18-20] and varicella zoster $[20,21]$ ) has been reported in several patients. Potentially lethal enteroviral encephalitis has been described after R-C in patients with ML [22-24]. Papovavirus infections have been linked to R-containing therapies in rare case reports. JC virus infection was reported in patients undergoing autologous hematopoietic stem cell transplantation soon after peri-transplant $R$ was added [25]. BK virus-associated leukoencephalopathy developed in a single patient soon after receiving $\mathrm{R}$, although this patient also had a complicated history of prior treatment for Hodgkin's disease [26]. Anecdotal reports have also indicated that Parvovirus B19 with pure red cell aplasia [27] and West Nile virus [28] may be linked to R-containing protocols.

$\mathrm{R}$ is also associated with impaired immunity against non-viral pathogens such as Babesia microti [29] and Pneumocystis jirovecii [30].

Infections are among the most important causes of morbidity and mortality in patients suffering from cancer. Although R-C is associated with a treatment outcome superior to standard $\mathrm{C}$, it is not clear whether or not the addition of $\mathrm{R}$ to standard $\mathrm{C}$ increases the risk of infections. The main objective of this review is to explore this. To do so we examine the evidence on infections from RCTs enrolling adults with CD20+ ML who undergo $\mathrm{R}-\mathrm{C}$ compared with identical $\mathrm{C}$ alone. Other endpoints included in the review are the risk of specific clinical presentation of infections, risk of death due to infection, risk of severe leucopenia, risk of severe granulocytopenia, risk of severe lymphopenia and overall response to therapy.

\section{Methods}

The review is reported according to the new "Preferred reporting items for systematic reviews and meta-analyses: the PRISMA statement" guidelines [31,32] (see Additional file 1).

\section{Eligibility criteria}

We included only peer-reviewed studies published in English, Spanish, French, German and Italian up to 31 July 2010.

The following inclusion criteria were used: being an RCT; enrolling adults with CD20+ ML; and identical C regimens in both arms with regard to type of $\operatorname{drug}(\mathrm{s})$, dosages and number of administrations (studies which included more than one $C$ regimen were excluded provided that disaggregated data for patients receiving the different Cs were available).

Exclusion criteria included the following: studies not reporting data about infection outcome; studies including non-lymphoma patients; studies without identical C in both arms; studies about maintenance purging and sequential treatment; studies without an R-free arm; non-randomized studies; studies including other MoAb in addition to or instead of R; studies in children (aged 16 or less); studies published in languages not mentioned above; and published editorials/reviews/letters/ comments.

\section{Information source and search strategy}

An electronic search was performed in three different databases (PubMed; Embase; Cochrane Controlled Trials Register) to reduce publication bias 
Different sets of key words, Mesh terms, truncation and filters for trials were used and the complete text for each string is provided in Additional file 2.

Further information was sought by e-mailing the authors to clarify uncertainty and omissions. In addition, we searched and analysed systematic reviews with similar inclusion criteria published over the last five years to determine if they reported additional studies or unpublished data. The references of all included studies and systematic reviews were also searched to find additional eligible studies

\section{Study selection}

Study selection was done by two independent reviewers (SL and AM). To guarantee transparency throughout the selection process, excluded papers were ranked according to the list of exclusion criteria. A single paper was either included or discarded if both reviewers had reached the same decision independently. For discordant decisions papers were assessed again by both reviewers and a consensus decision was taken.

\section{Data collection and data items}

Data about baseline population characteristics, type of intervention, outcome and study design were collected.

Population baseline data included the number of subjects randomized in each arm; participants' mean age in each arm; the proportion of different ML histological subtypes in each arm (this is according to WHO's "Classification of Tumours of Haematopoietic and Lymphoid Tissues [2]); line of treatment (that is, untreated or refractory); HIV status of participants; and HBV status of participants.

Intervention data included the type of drugs and dosage used in the $C$; the scheduled number of $C$ cycles; and the use of any other adjuvant therapies, such as antivirals, antibiotics, granulocyte colony-stimulating factor (G-CSF) or radiotherapy; dosage and number of $\mathrm{R}$ courses in the intervention arm.

Only the events which occurred during treatment were considered. Grades of adverse events were according to the NCI Common Terminology Criteria for Adverse Events Version 3.0 [33] and remission was defined according to the international definition for response criteria [34]. Outcome data were collected for: incidence of grade 3 and 4 infections [33] during treatment; incidence of different types of infections according to their clinical diagnosis, as reported by the authors; incidence of febrile neutropenia; the incidence of infection-related death; and the incidence of grade 3 or 4 leucopenia/granulocytopenia/lymphopenia.

We reviewed method(s) used to generate the allocation sequence, method(s) used to conceal the allocation sequence, measure(s) used to blind study participants and personnel from knowledge of which intervention a participant received, type of analysis, data about attrition, early stop of studies and study funding.

\section{Assessment of risk of bias}

To assess potential within-study risk of bias we considered several tools as reported below.

The methods used to generate the allocation sequence were adequate if authors referred to a random number table or stratification or a computer to generate a random number or minimization; according to Schulz et al. [35], we considered manual randomization, such as shuffling envelopes or throwing dice, inadequate.

The methods used to conceal allocation sequence to operator were adequate if authors referred to central allocation or sequentially numbered drug containers of identical appearance or sequentially numbered opaque sealed envelopes.

The methods used to blind study participants and personnel were adequate if the assessors of outcome and patients were blinded and an appropriate placebo, that is, an inactive intravenous (IV) compound in spite of R, was used.

The methods used to manage incomplete data reports and type of analysis were adequate if intent-to-treat analysis was undertaken without attrition or intent-to-treat analysis was undertaken and overall attrition was lower than $20 \%$ with similar attrition proportions in both arms.

The presence of potential conflict of interests was adequate if the study was mainly sponsored by government or any other organization without a direct link with $\mathrm{R}$ manufacturer and individual potential conflicting interests were disclosed.

The occurrence of early termination of the study was adequate if it did not occur.

A study was considered at low risk of bias if all the criteria were met and reported, at high risk of bias if one or more criteria were not met, or at unclear risk of bias if data for one or more items were not reported.

\section{Assessing the quality of a body of evidence}

We assessed the quality of the evidence supporting the meta-analysis results for each outcome according to the Grades of Recommendation, Assessment, Development and Evaluation Working Group approach (GRADE) as reported in the Cochrane Handbook for Systematic Reviews of Interventions [36,37].

In brief, the quality of evidence for each outcome was ranked according to four levels of quality; that is, high, moderate, low and very low. This was done with a twostep approach. First, we considered all outcomes as supported by high quality evidence since we included only RCTs. Then we downgraded the level of evidence 
according to four criteria: 1 . within-study risk of bias was present if less than two studies were at low risk of bias; 2 . imprecision of results was present if wide overall 95\% confidence interval (95\% CI) was present (according to GRADE guidance a confidence interval is wide when "the estimate is consistent with conflicting recommendations") [36]; 3. indirectness of evidence was present if indirect comparison between $\mathrm{R}-\mathrm{C}$ and $\mathrm{C}$ was done; and 4. risk of publication bias was present if funnel plots showed relevant asymmetry (see below).

The body of evidence was considered either of high quality (if no criterion was present), moderate quality (if one criterion was present), low quality (if two criteria were present) or very low quality (if three or more criteria were present).

\section{Statistical methods}

All outcomes were considered as binary variables. Risk ratio (RR) was used as a measure of association according to the formula:

$$
(\mathrm{Ai} / \mathrm{N} 1 \mathrm{i}) /(\mathrm{Ci} / \mathrm{N} 2 i)
$$

A fixed-effect model using the inverse-variance method was considered in all meta-analyses.

RR standard error (SE) was calculated according to the formula:

$$
S E\{\ln R R i\}=\operatorname{sqr}(1 / A i+1 / C i-1 / N 1 i-1 / N 2 i)
$$

Where sqr $=$ square root, $\mathrm{Ai}=$ events in the intervention arm in $\mathrm{i}^{\text {th }}$ study, $\mathrm{Ci}=$ events in the control arm in $\mathrm{i}^{\text {th }}$ study, N1 $\mathrm{i}=$ participants in intervention arm in $\mathrm{i}^{\text {th }}$ study and $\mathrm{N} 2 \mathrm{i}=$ participants in control arm in $\mathrm{i}^{\text {th }}$ study. Exponential form was always reported.

95\% CI were calculated according to the formula: 95\% $\mathrm{CI}=\mathrm{RR} \pm 1.96 \mathrm{SE}$

Meta-analysis was performed for outcomes reported in two or more studies. Meta-analyses of risk of infections, leucopenia and granulocytopenia included only studies reporting events according to the NCI grade (and only grade 3 and 4 events where considered for the analysis). Data about countable outcomes (that is, outcomes which may occur more than once in the same subject, such as infection, granulocytopenia, leucopenia and lymphopenia) were included in a meta-analysis only when patient level data (that is, risks or rates) were provided (we did not include studies reporting only the proportion of cycles in which events occurred).

In meta-analyses with at least four studies a funnel plot was generated and visually assessed by the help of Egger's graphical test $[38,39]$. We did not perform a formal statistical test for significance since the power and sensitivity of such tests is not well established when RR is used as the measure of association [37].
We considered heterogeneity negligible if I-squared was $<20.0 \%$, moderate if I-squared was between $20.0 \%$ and $49.9 \%$ and strong if I-squared was $\geq 50.0 \%$. For meta-analyses with moderate/strong heterogeneity, potential causes were explored by subgroup analysis for binary dichotomous covariates, that is: ML type (indolent/aggressive), line of treatment (untreated/refractory) and HIV status (positive/negative). A simple metaregression model was used for continuous covariates, that is: mean age and minimum/maximum number of $R$ cycles scheduled. In subgroup analyses with persisting heterogeneity, Der Simonian and Laird random-effect RR estimates were provided as terms of comparison. Chi-square $P$-value for heterogeneity was also provided in all fixed-effect models.

Sensitivity analysis was undertaken to evaluate the robustness of findings according to the relevant features which may affect within-study risk of bias (that is, type of funding; whether the study stopped early; methods used to manage incomplete data and randomization (this latter was adequate if both sequence generation and allocation concealment were adequate)).

STATA 11.1 (StataCorp Texas 77845 USA) package was used for the analysis and to generate forest plots and funnel plots.

\section{Results}

The literature search identified 729 unduplicated papers. Of these, 674 were excluded directly by reading the title and abstract and a further 39 were discarded after full evaluation of the text version. The remaining 16 papers comprising 17 RCTs for a total of 5,259 patients, were included (one paper, Pfreundschuh 2008, was divided into two RCTs according to the different $\mathrm{R}-\mathrm{C} / \mathrm{C}$ regimens). A summary table with the results of study selection, references of all included papers and the list of excluded papers are reported in Additional files 3, 4 and 5 respectively. All the data reported are those published, no additional data were provided by authors.

\section{Study characteristics}

Of the 17 RCTs, 16 were phase III RCTs to assess efficacy and 1 was a phase II RTC to assess toxicity.

Five RCTs enrolled 150 or less patients, six between 150 and 400 patients and six more than 400 patients. Mean age of participants was between 50.0 and 69.2 years (quartiles 55.5, 61.5, 68.0). Nine RCTs enrolled patients with indolent lymphoma (FC, MZL, CLL, ML and LPL) and eight RCTs enrolled patients with aggressive lymphoma (almost all DLBCL). Twelve RCTs enrolled only previously untreated subjects and five only refractory or relapsed patients. HIV status was reported in 13 studies (12 HIV negative and 1 HIV positive) and 4 studies did not explicitly report the HIV status of 
participants but it is likely they included only HIV negative subjects. HBV status was reported in seven studies, three RCTs included only HBsAg negative (the authors specified "absence of chronic hepatitis") and four RCTs included only anti-HBc negative (the authors specified "negative HBV serology"). Table 1 shows the baseline characteristics of the study populations.

Eight different protocols were associated with $\mathrm{R}$ as shown in Additional file 6. $\mathrm{R}$ was always administered at $375 \mathrm{mg} / \mathrm{m}^{2}$, given either the day before or the same day of $\mathrm{C}$. Number of $\mathrm{C}$ cycles, number of $\mathrm{R}$ administrations and other adjuvant therapies in each individual study are reported in Table 2. Minimum and maximum number of $\mathrm{R}$ cycles varies between three and eight (modal value six; median value six) and four and eight (modal value eight; median value six), respectively.

Data for binary outcomes (that is: overall response and deaths from infection) were reported as the number or proportion of patients experiencing the event. Countable outcomes data were reported in two different ways: 13 studies reported the number or proportion of patients experiencing at least one event throughout the therapy and 4 other studies analysed countable outcomes as the number or proportion of cycles of therapy in which events occurred at least once. No study clearly reported counts as rates or reported the absolute numbers of events and time at risk during therapy in either arm (see Additional file 7 for details about outcome reporting).

\section{Risk of bias within the studies}

All the studies were considered to be at high risk of bias. Figure 1 reports the summary of the within-study risk of bias analysis (Additional file 8 reports details of risk of bias analysis).

\section{Results of the meta-analyses}

Figure 2 shows the forest plots with results of the meta-analyses. In particular, pooled RRs did not indicate increased risk in patients receiving $\mathrm{R}-\mathrm{C}$ compared to those receiving $C$ for infections $(R R=1.00$; $C I 95 \%$ $=0.87$ to $1.14, P=0.943)$, risk of death as a consequence of infection $(\mathrm{RR}=1.60$; CI $95 \%=0.68$ to 3.75 , $P=0.279)$ and febrile neutropenia $(\mathrm{RR}=1.14 ; \mathrm{CI} 95 \%$ $=0.80$ to $1.63 ; P=0.478)$. In contrast, the pooled RRs for risk of leucopenia $(\mathrm{RR}=1.24$; CI $95 \%=1.12$ to 1.37; $P<0.001)$, granulocytopenia $(\mathrm{RR}=1.07$; $\mathrm{CI} 95 \%$ 1.02 to $1.12 ; P=0.008)$ and overall response $(1.12$, 95\% CI 1.09 to $1.15 P<0.001$ ) indicated that patients receiving $\mathrm{R}-\mathrm{C}$ had a greater risk of toxic effects, but a better end of treatment outcome, than patients in $C$ arm.

Heterogeneity was negligible (that is, $<20.0 \%$ ) in metaanalyses of risk of infection, risk of death as a consequence of infection and febrile neutropenia; moderate (that is, $20.0 \%$ to $49.9 \%$ ) in meta-analyses of leucopenia and granulocytopenia; and strong (that is, $>50 \%$ ) in the meta-analysis of overall response.

Table 1 Studies according to the study population baseline characteristics

\begin{tabular}{|c|c|c|c|c|c|c|c|}
\hline \multicolumn{8}{|c|}{ Characteristics of study populations } \\
\hline \multirow[t]{2}{*}{ Study } & \multicolumn{2}{|c|}{ Number ${ }^{a}$} & \multirow[t]{2}{*}{ Mean age } & \multirow[t]{2}{*}{ Line of treatment } & \multirow[t]{2}{*}{ Type of lymphoma } & \multirow[t]{2}{*}{ HIV status } & \multirow[t]{2}{*}{ HBV status } \\
\hline & Intervention & control & & & & & \\
\hline Aviles 2007a & 102 & 102 & 69.2 & untreated & aggressive & neg. & anti-HBC - \\
\hline Aviles 2007b & 98 & 98 & 59.7 & refractory & aggressive & neg. & anti-HBC - \\
\hline Aviles 2010 & 47 & 53 & 50.0 & refractory & aggressive & neg. & anti-HBC - \\
\hline Buske 2009 & 36 & 33 & 60.5 & untreated & indolent & neg.* & NR \\
\hline Coiffier 2002 & 202 & 197 & 69.0 & untreated & aggressive\# & neg. & $\mathrm{HBsAg}-$ \\
\hline Eve 2009 & 78 & 78 & 63.5 & untreated & indolent & neg. & anti-HBC - \\
\hline Forstpointner 2004 & 66 & 62 & 62.5 & refractory & indolent & neg.* & NR \\
\hline Habermann 2006 & 267 & 279 & 69.5 & untreated & aggressive & neg. & $N R$ \\
\hline Herold 2007 & 105 & 96 & 58.5 & untreated & indolent & neg. & $N R$ \\
\hline Hiddemann 2005 & 223 & 205 & 55.5 & untreated & indolent & neg.* & $N R$ \\
\hline Kaplan 2005 & 99 & 51 & 42.7 & untreated & aggressive & pos. & $N R$ \\
\hline Lenz 2005 & 62 & 60 & 61.5 & untreated & indolent & neg.* & $N R$ \\
\hline Marcus 2005 & 162 & 159 & 52.5 & untreated & Indolent § & neg. & HBsAg - \\
\hline Pfreundschuh 2008a & 304 & 305 & 68.5 & untreated & aggressive\# & neg. & NR \\
\hline Pfreundschuh 2008b & 306 & 307 & 68.0 & untreated & aggressive\# & neg. & NR \\
\hline Robak 2010 & 276 & 276 & 62.5 & refractory & indolent & neg. & HBsAg- \\
\hline van Oers 2006 & 234 & 231 & 54.5 & refractory & indolent & neg. & NR \\
\hline
\end{tabular}

a) number of patients included either in the control and intervention arm; NR = not reported; $\S$ this includes $1.2 \%$ undefined lymphomas; \# this includes 6 to $20 \%$ proportion of indolent or undefined; * HIV status not specified and presumed as negative. 
Table 2 Studies according to the type of intervention

\begin{tabular}{|c|c|c|c|c|c|c|c|}
\hline \multicolumn{8}{|c|}{ Intervention } \\
\hline \multirow[t]{2}{*}{ Study } & \multicolumn{3}{|c|}{ Intervention arm ${ }^{a}$} & \multicolumn{4}{|c|}{ Adjuvant therapies ${ }^{b}$} \\
\hline & Type of CHT & N. Cycles & R courses & G-CSF & Al prophylaxis & RTX & Other \\
\hline Aviles 2007a & CEOP & 6 & 6 & Discretionary & NR & No & No \\
\hline Aviles 2007b & CEOP & 6 & 6 & \multicolumn{4}{|c|}{ Not reported } \\
\hline Aviles 2010 & ESHAP & 6 & 6 & To all & No & No & No \\
\hline Buske 2009 & CHOP 21 & 4 to 8 & 4 to 8 & \multicolumn{4}{|c|}{ Not reported } \\
\hline Coiffier 2002 & CHOP 21 & 8 & 8 & Discretionary & $N R$ & No & No \\
\hline Eve 2009 & FC & 8 & 8 & NR & $P C P+V Z V$ & No & No \\
\hline Forstpointner 2004 & FCM & 4 & 4 & \multicolumn{4}{|c|}{ Not reported } \\
\hline Habermann 2006 & CHOP 21 & 6 to 8 & 4 to 5 & Discretionary & NR & No & M \\
\hline Herold 2007 & MCP & 8 & 8 & \multicolumn{4}{|c|}{ Not reported } \\
\hline Hiddemann 2005 & CHOP 21 & 6 to 8 & 6 to 8 & \multicolumn{4}{|c|}{ Not reported } \\
\hline Kaplan 2005 & CHOP 21 & 3 to 6 & 3 to 6 & To all & PCP & Discretionary & $M+A R T$ \\
\hline Lenz 2005 & CHOP 21 & 6 & 6 & \multicolumn{4}{|c|}{ Not reported } \\
\hline Marcus 2005 & CVP & 8 & 8 & \multicolumn{4}{|c|}{ Not reported } \\
\hline Pfreundschuh 2008a & CHOP 14 & 6 & 8 & To all & No & No & Pre-O \\
\hline Pfreundschuh 2008b & CHOP 14 & 8 & 8 & To all & No & No & Pre-O \\
\hline Robak 2010 & FC & 6 & 6 & Discretionary & No & No & No \\
\hline van Oers 2006 & CHOP 21 & 3 to 6 & 3 to 6 & \multicolumn{4}{|c|}{ Not reported } \\
\hline
\end{tabular}

Detailed protocol reported in additional file 6 . a) This describes the type of therapy for intervention arm (patients in control arm received the same therapy apart from rituximab); b) This includes all reported non randomized therapies. Al prophylaxis = anti-infective prophylaxis; ART = antiretroviral therapy; $\mathrm{C}=$ chemotherapy; - CSF = granulocyte colony stimulating factor; GRTX = radio therapy; $\mathrm{M}=$ meningeal prophylaxis with methotrexate; $\mathrm{N}$. cycles = number of cycles of scheduled therapy; NR = not reported; PCP = prophylaxis against $P$. jaroviecy; Pre-O = pre-treatment with single dose 1 mg vincristine and 100 mg prednisone orally for seven days; $\mathrm{R}$ course = number of cycles of scheduled rituximab course; VZV = prophylaxis against Varicella zoster virus

\section{Outcomes not included in the meta-analyses}

Patient-based risk of herpes zoster infections was reported in one study only (Coiffer 2002) that found 9/ 202 and 2/197 cases, in R-C and C arms respectively. Risk of hepatitis B was investigated in one study (Robak 2010) which reported $5 / 272$ cases in $\mathrm{R}-\mathrm{C}$ and $0 / 272$ in the $\mathrm{C}$ arm (this study included only patients without chronic hepatitis B). Cases of pneumonia were reported in one study (Robak 2010), which found 15/272 and 16/ 272 for R-C and C respectively.

One study (Aviles 2010) gave a detailed report of infections in the $\mathrm{R}-\mathrm{C}$ and $\mathrm{C}$ arms measured as the number of cycles in which at least one event occurred. This included: febrile neutropenia $(8 / 282$ and $12 / 318)$, sepsis (19/282 and 16/318), pneumonia (71/282 and 49/318), urinary infection $(12 / 282$ and $8 / 318)$, cytomegalovirus infection (28/282 and 3/318), herpes zoster (16/282 and $0 / 318)$, respiratory syncytial virus $(7 / 282$ and $0 / 318)$ and parvovirus (1/282 and 0/318).

Grade 3 and 4 lymphopenia was reported in one study (Fosterprint 2004) occurring in $51.2 \%$ and $39.4 \%$ of the $\mathrm{R}-\mathrm{C}$ and $\mathrm{C}$ arms respectively (the precise number of cycles in either arm was not given).

\section{Risk of bias across the studies}

Figure 3 shows the effect of publication bias. Overall the analysis showed that smaller studies tended to report higher overall response and lower risk of infections. Funnel plot asymmetry was mild for risk of infections (Figure 3A), suggesting low risk of publication bias, and substantial for leucopenia, (Figure 3B), granulocytopenia (Figure $3 \mathrm{C}$ ) and overall response (Figure 3D), suggesting a high risk of publication bias. However, since type of $M L$ is one of the major predictors of response to therapy we performed an additional analysis to evaluate plot asymmetry for overall response either in indolent (Figure $3 \mathrm{E}$ ) or aggressive (Figure $3 \mathrm{~F}$ ) lymphomas. In this way we found that asymmetry was greater for indolent lymphomas than for the aggressive lymphomas group.

Table 3 shows the results of the assessment of quality of evidence supporting each outcome according to the GRADE approach. The analysis indicates moderate to very low quality.

\section{Additional analysis}

Subgroup analysis according to the line of treatment (Figure 4A) explained the heterogeneity found in the meta-analysis for leucopenia. This analysis shows that the previously untreated subgroup had a homogenous and lower pooled RR (RR 1.20, 95\% CI 1.09 to 1.33 , Isquared $0 \%$ ) than the refractory subgroup (RR $2.0095 \%$ CI 1.34 to 2.99 , one study only). A partial explanation of heterogeneity in the overall response was given by subgroup analysis according to the type of ML (Figure 4B). 


\begin{tabular}{|llll}
\hline Study & &
\end{tabular}

Figure 1 Within-study risk of bias. This figure shows the summary of within-study risk of bias. Green icons indicate methodological features adequately undertaken and reported, yellow icons indicate methodological features unclearly reported, red icons indicate methodological features inadequately undertaken.

In the aggressive lymphomas subgroup we found a moderate heterogeneity within-group (I-squared 31.5\%, $P=$ 0.199), with minimal difference between fixed-effect and random-effect model estimates, while in the indolent lymphomas subgroup the heterogeneity remained strong (I-squared $70.5 \%, P=0.001$ ). Nevertheless, a beneficial effect was estimated by both fixed-effect and randomeffect models in the aggressive lymphomas subgroup (RR 1.07, 95\% CI 1.03 to 1.12 and RR 1.08 95\% CI 1.02 to 1.14 in fixed-effect and random-effect respectively) and indolent lymphomas subgroup (RR 1.15 95\% CI 1.11 to 1.19 and RR 1.24 95\% CI 1.13 to 1.37 in fixedeffect and random-effect respectively).

Results of the simple meta-regression model are provided in Table 4. Figure 4C shows the effect of mean age of participants on RR of granulocytopenia. The model indicates that $R R$ of granulocytopenia is estimated to be multiplied by 0.99 (0.98 to $1.00, P=$ 0.080) for each additional year of increase of the median age of study population (that is, the RR granulocytopenia of R-C vs. C is estimated to be multiplied by a factor $0.99^{10}=0.90$ for each 10 years of age increase of the study population). This model could explain all the heterogeneity found in the meta-analysis (residual heterogeneity $0 \%$ ). In addition since mean age seemed to explain the heterogeneity also for leucopenia (I-squared $=0 \%, P$ $=0.237$ ) which was already explained by line of treatment in subgroup analysis, we analysed the effect of mean age and line of treatment as concomitant covariates (using a bivariate meta-regression model). The results of this model are reported in Table 5 and show that line of treatment remained the best predictor of heterogeneity for severe leucopenia.

Meta-regression according to the minimum or number of $\mathrm{R}$ cycles did not provide additional evidence to explain heterogeneity (Table 4) and a similar result was obtained for the maximum or number of $\mathrm{R}$ cycles (data not shown)

Sensitivity analyses were conducted for all considered outcomes. These did not show any substantial difference between the pooled RRs in the overall meta-analyses and the meta-analyses of selected studies (complete results on sensitivity analysis, indicating only slight changes in leucopenia and granulocytopenia estimates, are reported in Additional file 9).

Table 6 shows the summary of evidence.

\section{Discussion}

This is the largest systematic review of infections in patients $(5,259)$ randomised to receive $C$ with or without $\mathrm{R}$ as part of induction protocol for CD20+ ML. Several relevant results emerged: 1 ). addition of $R$ to standard $C$ does not increase the global risk of infections nor does it increase the risk of lethal infections during therapy in patients with CD20+ ML; 2). there is a paucity of data about reactivation of latent viral pathogens which might be relevant in particular groups of patients (for example: HIV positive subjects and HBV carriers); 3). the addition of $\mathrm{R}$ to standard $\mathrm{C}$ can increase the risk of severe leucopenia and granulocytopenia during therapy in patients with CD20+ ML; and 4). compared to standard chemotherapy alone, $\mathrm{R}-\mathrm{C}$ increases the overall response both in indolent and aggressive lymphomas clinical variants.

Of the 10 studies included in the meta-analysis for global risk of infection, none found a significant increase of infections in the $\mathrm{R}-\mathrm{C}$ arm compared to the standard $\mathrm{C}$ arm, and one (Coiffer 2002) found a reduction ( $R R=$ $0.60,95 \%$ CI 0.38 to 0.96 ). The evidence for no increased risk is strong and is supported by low risk of publication bias, negligible heterogeneity in the meta- 


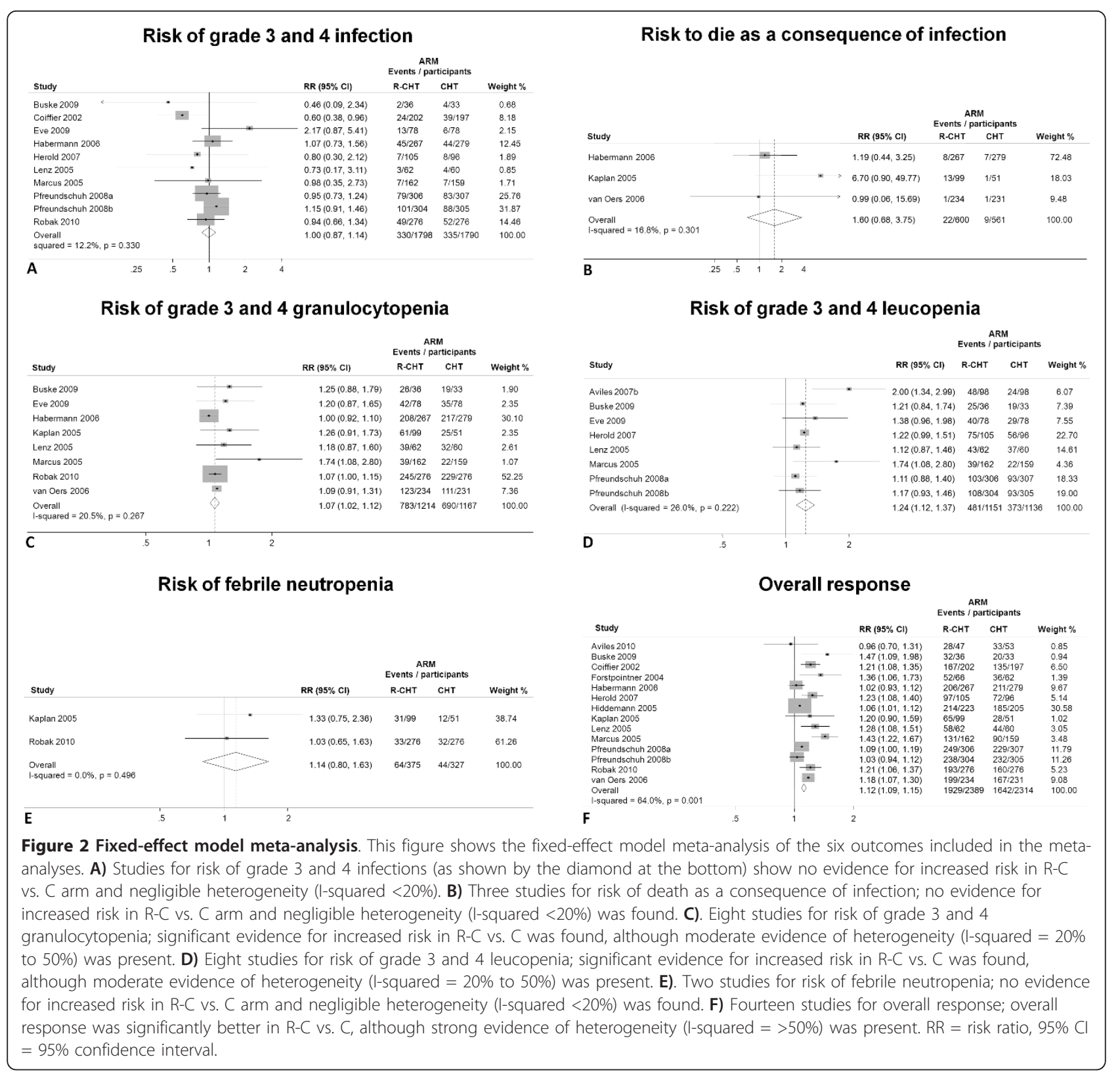

analysis and is consistent with indications from other systematic reviews $[4,5]$. However, the results may suffer from potential bias due to sub-optimal quality of the studies. In particular, the absence of proper allocation concealment in all the included studies is a relevant issue. Indeed, a proper blinding is not always achievable when frail subjects are to undergo parenteral drug therapies, as this would involve using an inactive IV infusion compound which may potentially expose patients in the control arm to additional (and perhaps, unjustified) risks. Although justified by ethical and practical reasons, the absence of proper blinding may bias results in two different ways. On one hand, the results may underestimate the actual RR. In fact, the awareness of patients' allocation may lead to systematically different and/or wider use of adjuvant treatments in the R-C arms (for example, use of anti-infective prophylaxis or G-CSF) than in the control arm. On the other hand, the results may overestimate the actual effect of $R$ on infection, as the consequence of the fact that patients in the intervention arm are more frequently exposed to IV procedures, such as $\mathrm{R}$ administrations.

The issue of whether or not the addition of $\mathrm{R}$ to standard $C$ may facilitate the reactivation of latent pathogens in selected groups of patients (for example, HIV positive subjects and HBV carriers) is more difficult to 

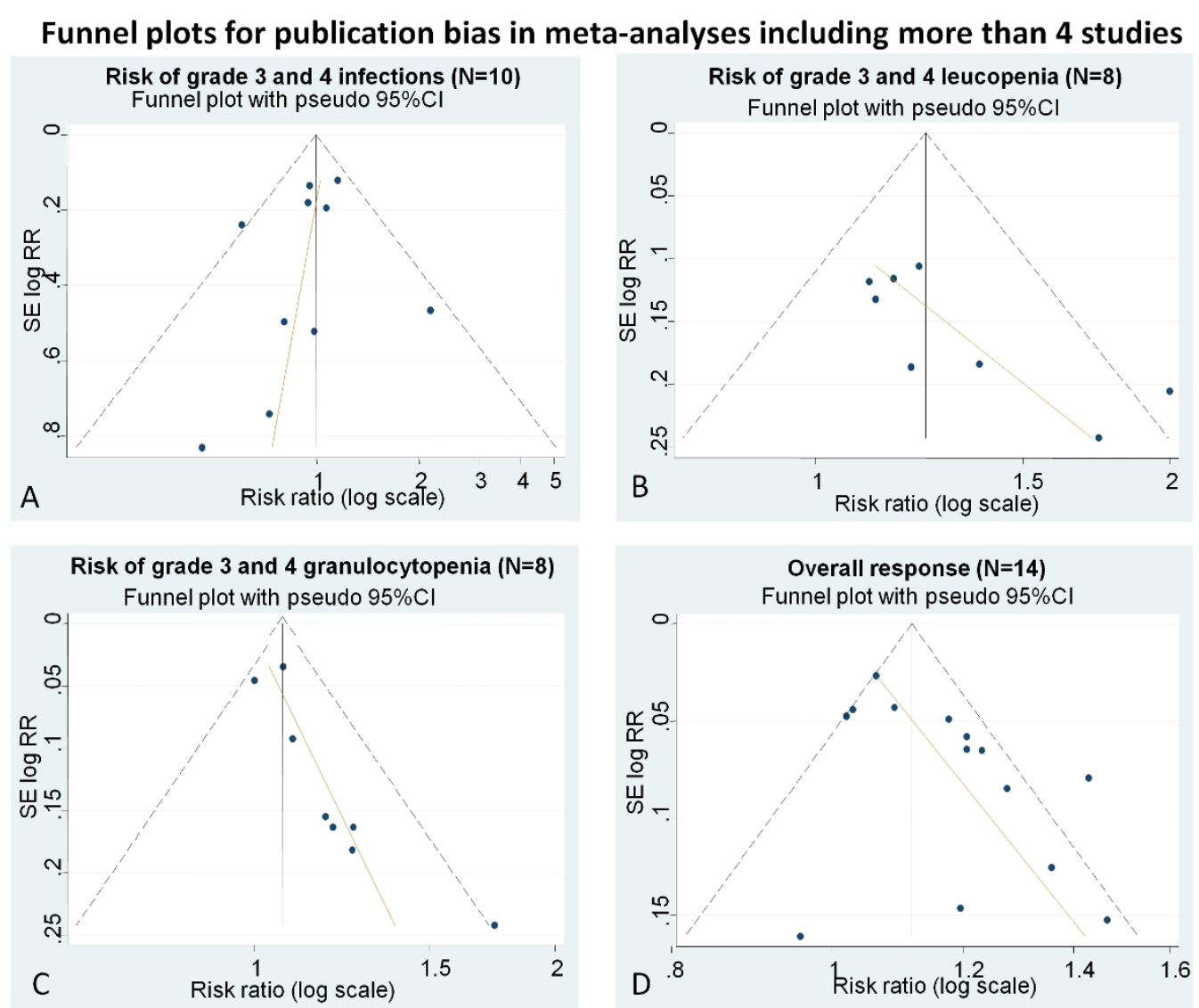

Funnel plots for publication bias according to the type of lymphoma
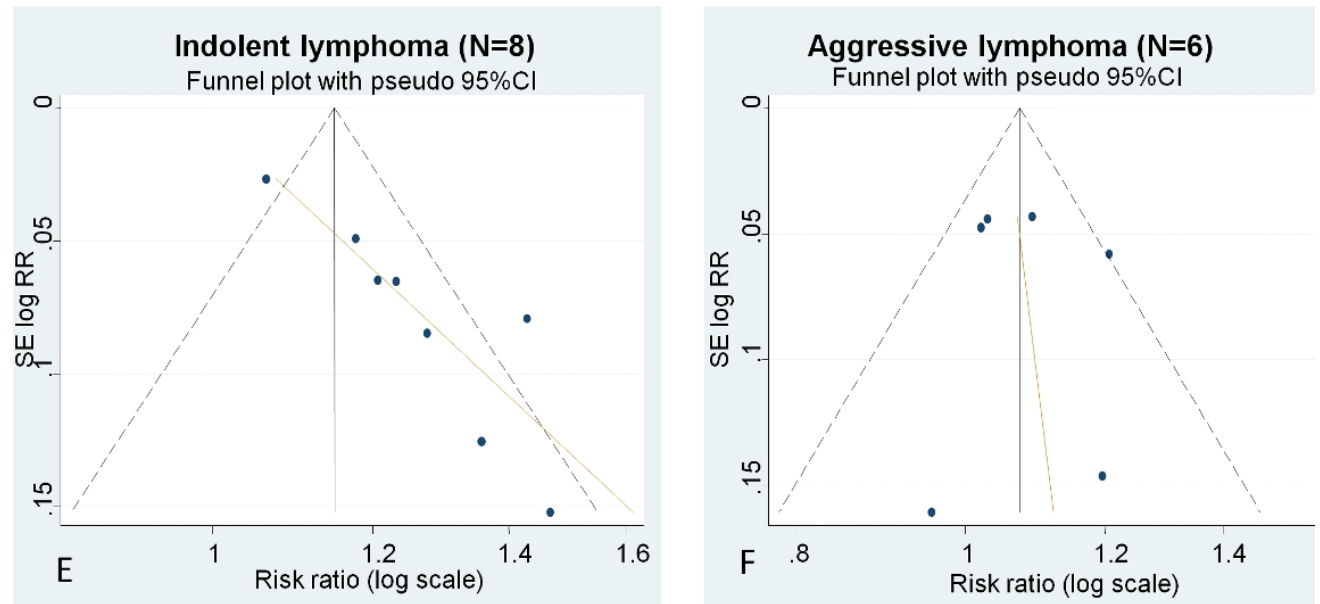

Figure 3 Funnel plots. This figure shows funnel plots produced using data from different meta-analyses including more than four studies. $X$ axes report risk ratio (RR) in the log scale; Y axes report the standard error of natural logarithm of risk ratio (SE log(RR)). The Egger's line (in orange) shows the degree of asymmetry. Relevant asymmetry, assessable by the degree of deviation of Egger's line indicates a high risk of potential publication bias. Asymmetry was negligible for risk of infection (A) and significant for risk of leucopenia (B) and risk of granulocytopenia (C). Asymmetry was also significant for overall response (D); however, funnel plots produced using data dividing studies according to the type of lymphoma (aggressive/indolent) showed persistently strong heterogeneity for indolent lymphomas (E) but negligible asymmetry for aggressive lymphomas (F). 
Table 3 Results of the assessment of quality of evidence

\begin{tabular}{|c|c|c|c|c|c|c|}
\hline \multicolumn{7}{|c|}{ Assessment of body of evidence supporting each outcome meta-analysis } \\
\hline \multicolumn{2}{|l|}{ Outcomes } & Within study risk of bias & Imprecision & Indirectness of evidence & $\begin{array}{l}\text { Funnel plot asymmetry } \\
\text { (Risk of pub bias) }\end{array}$ & Judgment \\
\hline \multicolumn{2}{|l|}{ Infections } & present & not present & not present & not present & Moderate \\
\hline \multicolumn{2}{|l|}{ Death for infection } & present & present & not present & not assessed & Very low \\
\hline \multicolumn{2}{|l|}{ Febrile neutropenia } & present & present & not present & not assessed & Very low \\
\hline \multicolumn{2}{|l|}{ Leucopenia } & present & not present & not present & present & Low \\
\hline \multicolumn{2}{|l|}{ Granulocytopenia } & present & not present & not present & present & Low \\
\hline \multirow[t]{3}{*}{ Overall response } & Overall & present & not present & not present & present & Low \\
\hline & Indolent & present & not present & not present & present & Low \\
\hline & Aggressive & present & not present & not present & not present & Moderate \\
\hline
\end{tabular}

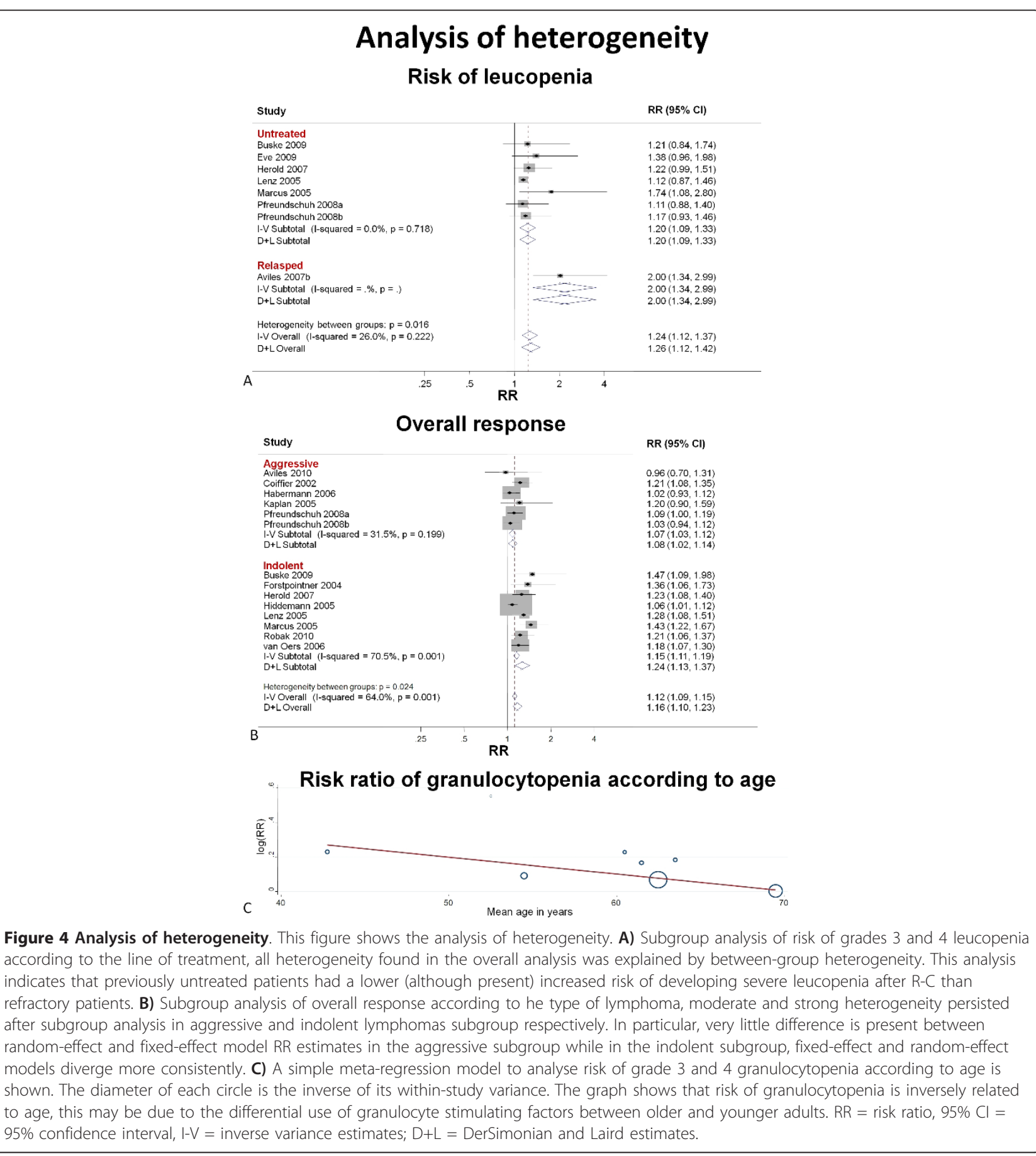


Table 4 The simple meta-regression analysis to explore heterogeneity for overall response, leucopenia and granulocytopenia according to continuous variables

\begin{tabular}{|c|c|c|c|c|c|c|c|c|c|}
\hline \multicolumn{10}{|c|}{ Meta-regression model } \\
\hline \multirow[t]{2}{*}{ Studies characteristics } & \multicolumn{3}{|c|}{ Leucopenia } & \multicolumn{3}{|c|}{ Granulocytopenia } & \multicolumn{3}{|c|}{ Overall response } \\
\hline & Res. & Coeff. $(95 \% \mathrm{Cl})$ & $p$ & Res. & Coeff. $(95 \% \mathrm{Cl})$ & $p$ & Res. & Coeff. $(95 \% \mathrm{Cl})$ & $\mathrm{p}$ \\
\hline Mean age & $0 \%$ & $\begin{array}{c}0.94 \\
(0.67 \text { to } 1.30)\end{array}$ & 0.237 & $0 \%$ & $\begin{array}{c}0.99 \\
(0.97 \text { to } 1.00)\end{array}$ & 0.080 & $64.7 \%$ & $\begin{array}{c}0.99 \\
\text { (0.98 to } 1.00)\end{array}$ & 0.271 \\
\hline Number of R cycles & $32.9 \%$ & $\begin{array}{c}0.95 \\
\text { (0.79 to } 1.15)\end{array}$ & 0.561 & $13.2 \%$ & $\begin{array}{c}1.03 \\
\text { (0.97 to } 1.08)\end{array}$ & 0.255 & $66.5 \%$ & $\begin{array}{c}1.01 \\
(0.97 \text { to } 1.05)\end{array}$ & 0.645 \\
\hline
\end{tabular}

The analysis indicates that mean age may explain heterogeneity found for granulocytopenia and leucopenia. Coeff. = regression coefficient in exponential form; Res. $=$ residual heterogeneity. $\mathrm{Cl}=$ confidence interval

examine for at least three reasons. Only one RCT (Kaplan 2005) included HIV positive patients and, although infections were accurately reported, we could not use them in the meta-analysis since disaggregate data of infections during therapy and during follow-up were not provided (it is noteworthy that this study found a statistically significant increase of risk of infection in the $\mathrm{R}-\mathrm{C}$ arm mainly due to patients with pretreatment CD4 counts less than 50 cells $/ \mathrm{ml}$ ). Second, only a few studies reported incident infections by aetiology. In particular, only one study systematically reported all infections by diagnosis (Aviles 2010) and three studies reported viral reactivation (Aviles 2010 and Coiffer 2002 herpetic infections; Robak 2010 HBV). These studies suggest that viral reactivations are more frequent in the $\mathrm{R}-\mathrm{C}$ arm which is consistent with data from a recent review including non-comparative studies [40], but we could not perform any specific analysis due to paucity of data. Third, RCTs selected only patients who were at very low or no risk of reactivation of relevant viral pathogens such as HBV.

The addition of $\mathrm{R}$ to standard $\mathrm{C}$ is associated with increased risk of severe leucopenia and granulocytopenia. Leucopenia and granulocytopenia were both reported in eight studies, both had a suggestion of publication bias and both showed moderate heterogeneity in overall meta-analysis that was eventually explained by

Table 5 The bivariate metaregression analysis to explore heterogeneity for leucopenia according mean age and line of treatment simultaneously

\begin{tabular}{lccc}
\hline \multicolumn{3}{c}{ Bivariate meta-regression model } \\
\hline Studies characteristics & \multicolumn{3}{c}{ Leucopenia } \\
\cline { 2 - 4 } & Res. & Coeff. (Cl 95\%) & $\boldsymbol{P}$ \\
\hline Mean age & $0 \%$ & 0.99 & 0.279 \\
Previous treatment & $(0.96$ to 1.01$)$ & \\
& & 1.59 & $\mathbf{0 . 0 8 4}$ \\
& & $\mathbf{0 . 9 1}$ to 2.78$)$ \\
\hline
\end{tabular}

The analysis confirms the results of subgroup analysis which indicates that the line of treatment is the best predictor of heterogeneity for severe leucopenia. Coeff. $=$ regression coefficient in exponential form; Res. = residual heterogeneity subgroup or meta-regression analysis. In particular, heterogeneity for leucopenia was due to one study (Aviles 2007b), which included refractory patients only. The subgroup analysis according to the line of treatment showed no within-group heterogeneity and a strong between-groups heterogeneity $(P=0.016)$. This effect may be explained by the more likely occurrence of leucopenia in frail patients undergoing aggressive therapy for refractory disease than in patients receiving first line treatment. The problem of heterogeneity for granulocytopenia is more complicated and this heterogeneity disappeared in meta-regression analysis according to the mean age of study population. The meta-regression indicates an inverse relation between mean age and RR (exponential regression coefficient $0.99,95 \%$ CI 0.98 to $1.00, P=0.080$ ). This result is at first sight anomalous since granulocytopenia might be expected to become more common with increasing age. However, we believe this could be explained by a greater use of G-CSF in RCTs enrolling older patients. G-CSF was mainly given according to clinical judgment, so it is possible that clinicians were more likely to give G-CSF to older patients. Published studies suggest that the use of G-CSF is beneficial in elderly patients with cancer [41] and in those receiving R-containing therapies [42].

Finally, the results of meta-analysis on efficacy were consistent with other reviews focused on this issue $[4,5]$ which have shown that $\mathrm{R}-\mathrm{C}$ increases the response to therapy in patients with $\mathrm{ML}$ and that this effect is more evident in indolent than in aggressive lymphoma.

Several limitations may affect the result of the present study. First, the pooled RR for overall response in indolent ML may be overestimated as a result of publication bias. In fact, smaller studies suggest a more beneficial effect of $\mathrm{R}$ than larger ones (asymmetry of funnel plot) and small negative studies are less likely to be published than large negative ones. Therefore, we may have missed a number of small negative studies. Second, real associations between R-C and countable outcomes (that is, infections, leucopenia, granulocytopenia and febrile neutropenia) may be misinterpreted. In fact, we could not obtain additional data from authors about the rates of 
Table 6 The overall results of the study

\begin{tabular}{|c|c|c|c|c|c|c|c|}
\hline \multicolumn{8}{|c|}{ Summary of evidence } \\
\hline \multicolumn{2}{|l|}{ Outcomes } & $\begin{array}{c}\text { Risk } \\
\text { ratio } \\
(95 \% \\
\mathrm{Cl})\end{array}$ & $P$ & $\begin{array}{l}\text { Num. } \\
\text { studies }\end{array}$ & $\begin{array}{l}\text { Num. of } \\
\text { participants }\end{array}$ & $\begin{array}{l}\text { Quality of } \\
\text { evidence }\end{array}$ & Heterogeneity (H) \\
\hline \multicolumn{2}{|l|}{ Infections } & $\begin{array}{c}1.00 \\
(0.87 \\
\text { to } \\
1.14)\end{array}$ & 0.943 & 10 & 3,585 & Moderate & $\mathrm{I}^{2}<20 \%$ not further investigated \\
\hline \multicolumn{2}{|c|}{ Death from infection } & $\begin{array}{c}1.60 \\
(0.68 \\
\text { to } \\
3.75)\end{array}$ & 0.279 & 3 & 1,161 & Very Low & $\mathrm{I}^{2}<20 \%$ not further investigated \\
\hline \multicolumn{2}{|c|}{ Febrile neutropenia } & $\begin{array}{c}1.14 \\
(0.80 \\
\text { to } \\
1.63)\end{array}$ & 0.496 & 2 & 702 & Very Low & None \\
\hline \multirow[t]{3}{*}{ Leucopenia } & Overall & $\begin{array}{c}1.24 \\
(1.12 \\
\text { to } \\
1.37)\end{array}$ & $>0.001$ & 8 & 2287 & Low & $\begin{array}{c}\text { No within group } \mathrm{H} \text { after subgroup analysis for line of treatment, } \\
\text { between group } \mathrm{HP}=0.016\end{array}$ \\
\hline & Untreated & $\begin{array}{c}1.20 \\
(1.09 \\
\text { to } \\
1.33)\end{array}$ & $>0.001$ & 7 & 2,091 & & \\
\hline & Refractory & $\begin{array}{c}2.00 \\
(1.34 \\
\text { to } \\
2.99)\end{array}$ & 0.001 & 1 & 196 & & \\
\hline \multicolumn{2}{|c|}{ Granulocytopenia } & $\begin{array}{c}1.07 \\
(1.02 \\
\text { to } \\
1.12)\end{array}$ & 0.008 & 8 & 2,381 & Low & $\mathrm{H}$ disappears in metaregression according to mean age \\
\hline \multirow[t]{3}{*}{$\begin{array}{l}\text { Overall } \\
\text { response }\end{array}$} & Overall & $\begin{array}{c}1.12 \\
(1.09 \\
\text { to } \\
1.15)\end{array}$ & $>0.001$ & 14 & 4,703 & Low & $\begin{array}{l}\text { Persistent strong } \mathrm{H} \text { in indolent lymphomas and moderate } \mathrm{H} \text { in } \\
\text { aggressive lymphomas after subgroup and metaregression analyses. }\end{array}$ \\
\hline & $\begin{array}{l}\text { Indolent } \\
\text { lymphomas }\end{array}$ & $\begin{array}{c}1.15 \\
(1.11 \\
\text { to } \\
1.19)\end{array}$ & $>0.001$ & 6 & 2,417 & Low & \\
\hline & $\begin{array}{l}\text { Aggressive } \\
\text { lymphomas }\end{array}$ & $\begin{array}{c}1.07 \\
(1.03 \\
\text { to } \\
1.12)\end{array}$ & 0.002 & 8 & 2,286 & Moderate & \\
\hline
\end{tabular}

these events, and, basing these meta-analyses on published data only, we used the risk (number of patients with at least one event over overall exposed at risk) as the measure of association. The degree and direction of this potential bias is not predictable. Third, the choice of dividing all ML into only aggressive and indolent clinical type (due to a lack of disaggregated outcomes data by histology of different indolent ML) may be too simplistic and it may explain the strong heterogeneity found in the overall response meta-analysis of indolent clinical type. Fourth, we considered only grade 3 and 4 infections, which occurred during induction therapy; therefore, we did not estimate the potential risk of late onset infections, the potential increased risk of infection in patients who eventually undergo maintenance therapy and the actual risk of mild and moderate infection (it is noteworthy that there is evidence of increased risk of infections when long lasting Rbased regimens, such as maintenance protocols, are undertaken $[10,43])$. Fifth, the quality of all the meta-analyses might be affected by the sub-optimal quality of the studies. In particular, none of the studies was blinded and patients underwent a number of unrestricted adjuvant therapies such as use of anti-infective agents and G-CSF. Sixth, due to lack of published data, we could not definitively determine whether or not the addition of $\mathrm{R}$ to standard $\mathrm{C}$ may facilitate the reactivation of latent pathogens. Finally, we 
did not include two papers written in Chinese. We could not retrieve the authors' e-mail addresses nor were we able to obtain the full-text copy of the papers in the original language to be translated. Although such as exclusion might potentially bias our results, we do not believe this will affect the general application of our findings.

\section{Conclusions}

In conclusion we find that $\mathrm{R}-\mathrm{C}$ is superior to standard $\mathrm{C}$ in terms of overall response in patients affected from CD20 positive ML without increasing the incidence of severe infections. In addition, overall survival has been improved in many of the included studies as also reported in recent systematic reviews focused on $\mathrm{R}$ efficacy in induction treatment of CD20+ ML [4-6]. Nevertheless, data on patients with overt and/or latent viral infections are lacking and in our opinion, more studies are needed to explore the potential effect of $\mathrm{R}$ on latent viral infections (for example, herpesvirus and HBV infections) and on selected groups of patients (for example, patients with advanced HIV infection, anti-HBc positive and/or HBsAg positive patients). Future studies should preferably be RCTs or, if RCTs are considered inappropriate, they should be large and methodologically sound observational ones (such as prospective cohort studies). To increase the quality of future systematic reviews, new studies should provide the overall number of countable outcomes along with the time at risk.

\section{Additional material}

Additional file 1: PRISMA check list. PRISMA's items and their
application within the paper.
Additional file 2: Strings. Strings used to perform the search.
Additional file 3: Paper selection. Summary of paper selection results.
Additional file 4: Included papers. Bibliography of included papers
sorted by author's name.
Additional file 5: Excluded papers. List of excluded papers with
exclusion criteria sorted by year of publication.
Additional file 6: Protocols. Details of therapies in each different RTC.
Additional file 7: Reported outcome. Details of studied outcomes as
reported in each different RTC.
Additional file 8: Within study risk of bias. Details of the studies
according to within risk of bias of each different RTC.
Additional file 9: Sensitivity analysis. Summary of complete results of
sensitivity analysis.

\footnotetext{
Abbreviations

95\% Cl: 95\% confidence interval; Anti-HBc: anti hepatitis B virus core antigen antibodies; Anti-HBs: anti hepatitis B virus surface antigen antibodies; CEOP: cyclophosphamide, epirubicin, vincristine and prednisone (each cycle given every 21 days); CHOP 14: cyclophosphamide, doxorubicin, vincristine and prednisone (each cycle given every 14 days); CHOP 21: cyclophosphamide, doxorubicin, vincristine and prednisone (each cycle given every 21 days); C: chemotherapy; CLL: chronic lymphocytic leukemia/small lymphocytic lymphoma; CVP: cyclophosphamide, vincristine and prednisone (each cycle
}

given every 14 days); DLBCL: diffuse large B cell lymphoma; ESHAP: etoposide, methylprednisolone, cytarabine and cisplatin (each cycle every 28 days); FC: cyclophosphamide and fludarabine (each cycle given every 28 days); FCM: cyclophosphamide, fludarabine and mitoxantrone (each cycle given every 28 days); FL: follicular lymphoma; G-CSF: granulocyte colony stimulating factor; GRADE: Grades of Recommendation, Assessment, Development and Evaluation Working Group; HBsAg: hepatitis B virus surface antigen; HBV: hepatitis B virus; HIV: human immunodeficiency virus; IV: intra-venous; LPL: Iymphoplasmacytic lymphoma; MCP: mitoxantrone, chlorambucil and prednisone (each cycle given every 28 days); ML: malignant lymphoma; MoAb: monoclonal antibody; MZL: marginal zone lymphoma; NCl: US National Cancer Institute; P: P-value; PRISMA: Preferred reporting items for systematic reviews and meta-analyses; $R$ : rituximab; $R-C$ : chemotherapy plus rituximab; RCT: randomized controlled trial; RR: risk ratio.

\section{Acknowledgements}

This study has been funded by the Italian Society for Infection Disease and Tropical Medicine (SIMIT).

\section{Author details}

${ }^{1}$ National Institute for Infectious Diseases, INMI-Lazzaro Spallanzani Via Portuense, 29200149 Rome, Italy. ${ }^{2}$ Department of Medical Microbiology, Royal Free Hospital, Pond Street, London NW3 2QG, UK. ${ }^{3}$ London School of Hygiene and Tropical Medicine, Keppel Street, London WC1E 7HT, UK. ${ }^{4}$ Department of Haematology, Royal Free Hospital, Pond Street, London NW3 2QG, UK.

\section{Authors' contributions}

SL designed the study, set up the Epi-Info databases, defined the search strings, performed the literature search, selected papers, assessed papers, extracted data, made the analysis, interpreted results and drafted the text. ACM performed the literature search, selected papers, assessed papers, extracted data, interpreted results, and reviewed and approved the final text. PEF, AGP and GI interpreted results, and reviewed and approved the final text. CK approved the study design, interpreted results, reviewed the text and gave final approval to the paper.

\section{Competing interests}

The authors declare that they have no competing interests.

Received: 12 January 2011 Accepted: 12 April 2011

Published: 12 April 2011

\section{References}

1. Sant M, Allemani C, Tereanu C, De Angelis R, Capocaccia R, Visser O, Marcos-Gragera R, Maynadié M, Simonetti A, Lutz JM, Berrino F: Incidence of hematological malignancies in Europe by morphological subtype: results of the HAEMACARE project. Blood 2010, 116:3724-3734.

2. Jaffe ES, Harris NL, Stein H, Isaacson PG: Classification of lymphoid neoplasms: the microscope as a tool for disease discovery. Blood 2008, 112:4384-4399.

3. Coiffier B: Treatment of non-Hodgkin's lymphoma: a look over the past decade. Clin Lymphoma Myeloma 2006, 7:57-13.

4. Schulz H, Bohlius J, Skoetz N, Trelle S, Kober T, Reiser M, Dreyling M, Herold M, Schwarzer G, Hallek M, Engert A: Chemotherapy plus Rituximab versus chemotherapy alone for B-cell non-Hodgkin's lymphoma. Cochrane Database Syst Rev 2007, CD003805.

5. Gao G, Liang X, Jiang J, Zhou X, Huang R, Chu Z, Zhan Q: A systematic review and meta-analysis of immunochemotherapy with rituximab for B-cell non-Hodgkin's lymphoma. Acta Oncol 2010, 49:3-12.

6. Fang $C, X u$ W, Li JY: A systematic review and meta-analysis of rituximabbased mimmunochemotherapy for subtypes of diffuse large B cell lymphoma. Ann Hematol 2010, 89:1107-1113.

7. Rafailidis PI, Kakisi OK, Vardakas K, Falagas ME: Infectious complications of monoclonal antibodies used in cancer therapy: a systematic review of the evidence from randomized controlled trials. Cancer 2007, 109:2182-2189.

8. Kaplan LD, Lee JY, Ambinder RF, Sparano JA, Cesarman E, Chadburn A, Levine AM, Scadden DT: Rituximab does not improve clinical outcome in a randomized phase 3 trial of $\mathrm{CHOP}$ with or without rituximab in patients with HIV-associated non-Hodgkin lymphoma: AIDS-Malignancies Consortium Trial 010. Blood 2005, 106:1538-1543. 
9. Spina M, Jaeger U, Sparano JA, Talamini R, Simonelli C, Michieli M, Rossi G, Nigra E, Berretta M, Cattaneo C, Rieger AC, Vaccher E, Tirelli U: Rituximab plus infusional cyclophosphamide, doxorubicin, and etoposide in HIVassociated non-Hodgkin lymphoma: pooled results from 3 phase 2 trials. Blood 2005, 105:1891-1897.

10. Vidal L, Gafter-Gvili A, Leibovici L, Dreyling M, Ghielmini M, Hsu Schmitz SF, Cohen A, Shpilberg O: Rituximab maintenance for the treatment of patients with follicular lymphoma: systematic review and meta-analysis of randomized trials. J Natl Cancer Inst 2009, 101:248-255.

11. Aksoy S, Harputluoglu H, Kilickap S, Dede DS, Dizdar O, Altundag K, Barista I: Rituximab-related viral infections in lymphoma patients. Leuk Lymphoma 2007, 48:1307-1312.

12. Targhetta C, Cabras MG, Mamusa AM, Mascia G, Angelucci E: Hepatitis B virus-related liver disease in isolated anti-hepatitis B-core positive lymphoma patients receiving chemo- or chemo-immune therapy. Haematologica 2008, 93:951-952.

13. Niscola P, Del Principe MI, Maurillo L, Venditti A, Buccisano F, Piccioni D, Amadori S, Del Poeta G: Fulminant B hepatitis in a surface antigennegative patient with B-cell chronic lymphocytic leukaemia after rituximab therapy. Leukemia 2005, 19:1840-1841.

14. Perceau G, Diris N, Estines O, Derancourt C, Lévy S, Bernard P: Late lethal hepatitis $B$ virus reactivation after rituximab treatment of low-grade cutaneous B-cell lymphoma. Br J Dermatol 2006, 155:1053-1056.

15. Dervite I, Hober D, Morel P: Acute hepatitis $B$ in a patient with antibodies to hepatitis $B$ surface antigen who was receiving rituximab. N Engl J Med 2001, 344:68-69.

16. Tsutsumi Y, Tanaka J, Kawamura T, Miura T, Kanamori H, Obara S, Asaka M, Imamura M, Masauzi N: Possible efficacy of lamivudine treatment to prevent hepatitis $B$ virus reactivation due to rituximab therapy in a patient with non-Hodgkin's lymphoma. Ann Hematol 2004, 83:58-60.

17. Hamaki T, Kami M, Kusumi E, Ueyama J, Miyakoshi S, Morinaga S, Mutou Y: Prophylaxis of hepatitis $B$ reactivation using lamivudine in a patient receiving rituximab. Am J Hematol 2001, 68:292-294

18. Suzan F, Ammor M, Ribrag V: Fatal reactivation of cytomegalovirus infection after use of rituximab for a post-transplantation lymphoproliferative disorder. N Engl J Med 2001, 345:1000

19. Vallet S, Tempescul A, Tran A, Legrand-Quillien MC, Narbonne V, Berthou C: Cytomegalovirus-associated meningoradiculoneuritis after treatment of mantle cell lymphoma with a combination of chemotherapy and rituximab. Ann Hematol 2005, 84:545-547.

20. Bermúdez A, Marco F, Conde E, Mazo E, Recio M, Zubizarreta A: Fatal visceral varicella-zoster infection following rituximab and chemotherapy treatment in a patient with follicular lymphoma. Haematologica 2000, 85:894-895.

21. Precupanu CM, Girodet J, Mariani P, Zanni M, Mathiot C, Escande MC, Brault P, Decaudin D: Pseudo-bowel obstruction due to varicella zoster virus infection after autologous stem cell transplantation. Am J Hematol 2009, 84:127-128.

22. Quartier $P$, Tournilhac $O$, Archimbaud C, Lazaro L, Chaleteix C, Millet $P$, Peigue-Lafeuille H, Blanche S, Fischer A, Casanova JL, Travade P, Tardieu M Enteroviral meningoencephalitis after anti-CD20 (rituximab) treatment. Clin Infect Dis 2003, 36:e47-49.

23. Archimbaud C, Bailly JL, Chambon M, Tournilhac O, Travade P, PeigueLafeuille $\mathrm{H}$ : Molecular evidence of persistent echovirus 13 meningoencephalitis in a patient with relapsed lymphoma after an outbreak of meningitis in 2000. J Clin Microbiol 2003, 41:4605-4610.

24. Padate BP, Keidan J: Enteroviral meningoencephalitis in a patient with non-Hodgkin's lymphoma treated previously with rituximab. Clin Lab Haematol 2006, 28:69-71

25. Goldberg SL, Pecora AL, Alter RS, Kroll MS, Rowley SD, Waintraub SE, Imrit K, Preti RA: Unusual viral infections (progressive multifocal leukoencephalopathy and cytomegalovirus disease) after high-dose chemotherapy with autologous blood stem cell rescue and peritransplantation rituximab. Blood 2002, 99:1486-1488.

26. Matteucci P, Magni M, Di Nicola M, Carlo-Stella C, Uberti C, Gianni AM: Leukoencephalopathy and papovavirus infection after treatment with chemotherapy and anti-CD20 monoclonal antibody. Blood 2002, 100:1104-1105.

27. Song KW, Mollee P, Patterson B, Brien W, Crump M: Pure red cell aplasia due to parvovirus following treatment with $\mathrm{CHOP}$ and rituximab for $\mathrm{B}$ cell lymphoma. Br J Haematol 2002, 119:125-127.
28. Mawhorter SD, Sierk A, Staugaitis SM, Avery RK, Sobecks R, Prayson RA, Procop GW, Yen-Lieberman B: Fatal West Nile Virus infection after rituximab/fludarabine-induced remission for non-Hodgkin's lymphoma. Clin Lymphoma Myeloma 2005, 6:248-250.

29. Krause PJ, Gewurz BE, Hill D, Marty FM, Vannier E, Foppa IM, Furman RR Neuhaus E, Skowron G, Gupta S, McCalla C, Pesanti EL, Young M, Heiman D, Hsue G, Gelfand JA, Wormser GP, Dickason J, Bia FJ, Hartman B, Telford SR, Christianson D, Dardick K, Coleman M, Girotto JE, Spielman A: Persistent and relapsing babesiosis in immunocompromised patients. Clin Infect Dis 2008, 46:370-376.

30. Ennishi D, Terui Y, Yokoyama M, Mishima Y, Takahashi S, Takeuchi K, Ikeda K, Tanimoto M, Hatake K: Increased incidence of interstitial pneumonia by CHOP combined with rituximab. Int J Hematol 2008, 87:393-397.

31. Moher D, Liberati A, Tetzlaff J, Altman DG, PRISMA Group: Preferred reporting items for systematic reviews and meta-analyses: the PRISMA statement. BMJ 2009, 339:b2535.

32. Liberati A, Altman DG, Tetzlaff J, Mulrow C, Gøtzsche PC, loannidis JP, Clarke M, Devereaux PJ, Kleijnen J, Moher D: The PRISMA statement for reporting systematic reviews and meta-analyses of studies that evaluate healthcare interventions: explanation and elaboration. BMJ 2009, 339: b2700.

33. US National Institute of Health - National Cancer Institute Cancer Therapy Evaluation Program, Common Terminology Criteria for Adverse Events, Version 3.0, DCTD, NCl, NIH, DHHS. 2006 [http://ctep.cancer.gov].

34. Cheson BD, Horning SJ, Coiffier B, Shipp MA, Fisher RI, Connors JM, Lister TA, Vose J, Grillo-López A, Hagenbeek A, Cabanillas F, Klippensten D, Hiddemann W, Castellino R, Harris NL, Armitage JO, Carter W, Hoppe R, Canellos GP: Report of an international workshop to standardize response criteria for non-Hodgkin's lymphomas. $\mathrm{NCI}$ Sponsored International Working Group. J Clin Oncol 1999, 17:1244.

35. Schulz KF, Grimes DA: Generation of allocation sequences in randomised trials: chance, not choice. Lancet 2002, 359:515-519.

36. Atkins $D$, Best $D$, Briss PA, Eccles $M$, Falck-Ytter $Y$, Flottorp $S$, Guyatt GH, Harbour RT, Haugh MC, Henry D, Hill S, Jaeschke R, Leng G, Liberati A, Magrini N, Mason J, Middleton P, Mrukowicz J, O'Connell D, Oxman AD, Phillips B, Schünemann HJ, Edejer TT, Varonen H, Vist GE, Williams JW Jr, Zaza S, GRADE Working Group: Grading quality of evidence and strength of recommendations. BMJ 2004, 328:1490.

37. In Cochrane Handbook for Systematic Reviews of Interventions Version 5.0.2 (updated September 2009). Edited by: Higgins JPT, Green S. The Cochrane Collaboration; 2009:[http://www.cochrane-handbook.org].

38. Egger M, Davey Smith G, Schneider M, Minder C: Bias in meta-analysis detected by a simple, graphical test. BMJ 1997, 315:629-634.

39. Sterne JAC, Harbord RM: Funnel plots in meta-analysis. Stata Journal 2004, 4:127-141[http://www.stata-journal.com/sjpdf.html?articlenum=st0061].

40. Gea-Banacloche JC: Rituximab-associated infections. Semin Hematol 2010, 47:187-198.

41. Lyman GH, Kuderer N, Agboola O, Balducci L: Evidence-based use of colony-stimulating factors in elderly cancer patients. Cancer Control 2003, 10:487-499.

42. Cartron G, Zhao-Yang L, Baudard M, Kanouni T, Rouillé V, Quittet P, Klein B, Rossi JF: Granulocyte-macrophage colony-stimulating factor potentiates rituximab in patients with relapsed follicular lymphoma: results of a phase II study. J Clin Oncol 2008, 26:2725-2771.

43. Salles G, Seymour JF, Offner F, López-Guillermo A, Belada D, Xerri L, Feugier P, Bouabdallah R, Catalano JV, Brice P, Caballero D, Haioun C, Pedersen LM, Delmer A, Simpson D, Leppa S, Soubeyran P, Hagenbeek A, Casasnovas $O$, Intragumtornchai T, Fermé C, da Silva MG, Sebban C, Lister A, Estell JA, Milonen G, Sonet A, Mendila M, Coiffier B, Tilly H: Rituximab maintenance for 2 years in patients with high tumour burden follicular lymphoma responding to rituximab plus chemotherapy (PRIMA): a phase 3, randomised controlled trial. Lancet 2011, 377:42-51.

\section{Pre-publication history}

The pre-publication history for this paper can be accessed here: http://www.biomedcentral.com/1741-7015/9/36/prepub

doi:10.1186/1741-7015-9-36

Cite this article as: Lanini et al:: Risk of infection in patients with lymphoma receiving rituximab: systematic review and meta-analysis. BMC Medicine 2011 9:36. 